\title{
Nivel de conocimiento y la práctica del cuidado de la vía aérea que realizan las enfermeras en pacientes críticos con tubo orotraqueal en la Clínica Good Hope, Lima
}

\author{
Bertha Liz Gamboa-Neyre ${ }^{1 *}$, Héctor Huamanchoque-Chuctaya ${ }^{2}$, Milagros Del Pilar Ramos-Quispe ${ }^{3}$ \\ ${ }^{1}$ Clínica Good Hope - Malecón Balta 956, Miraflores - Lima, Perú \\ ${ }_{2}^{2}$ Hospital de Baja Complejidad Vitarte - Av. Nicolás Ayllón 5880 - ATE. \\ ${ }^{3}$ Puesto de Salud Horacio Zevallos -Av. J. Zubieta S/N. AA.HH. Horacio Zevallos
}

\begin{abstract}
RESUMEN
Objetivo: Determinar el nivel de conocimiento y la práctica del cuidado de la vía aérea que realizan las enfermeras en pacientes críticos con tubo orotraqueal en la Clínica Good Hope. Lima, febrero -junio 2012. Material y Métodos: El estudio realizado fue observacional, descriptivo, de corte transversal y diseño correlacional. La población estuvo conformada por 30 enfermeras. Para el nivel de conocimiento se utilizó un cuestionario de 20 preguntas y para la práctica se utilizó una lista de cotejo de 25 criterios. La prueba de confiabilidad se estableció según el Coeficiente alfa de Cronbach $(0,87)$. Resultados: Del 100\% de las enfermeras de la muestra, se evidenció que el $50 \%$ tuvo un nivel excelente de conocimientos acerca del cuidado de la vía aérea. La práctica del cuidado de la vía aérea fue, del mismo modo, de nivel excelente en un 50\%. Conclusiones: Según la prueba estadística del Chi cuadrado de Pearson, se encontró relación significativa entre el nivel de conocimiento y la práctica del cuidado de la vía aérea que realizan las enfermeras en pacientes críticos con tubo orotraqueal en la Clínica Good Hope (valor $p=, 000$ ).
\end{abstract}

Palabras clave: Factor de riesgo, retención urinaria, post operado inmediato.

\begin{abstract}
Objective: Determine the level of knowledge and practice of airway care performed by nurses in critical patients with endotracheal tube in Good Hope Clinic. Lima, February to June 2012. Materials and Methods: The study was observational, descriptive, cross-sectional and correlational design. The population consisted of 30 nurses. To the knowledge level, a questionnaire of 20 questions and the practice was used a checklist of 25 criteria. Reliability test was 0,873 according to the Cronbach alpha coefficient. Results: $100 \%$ of the nurses in the sample showed that $50 \%$ had an excellent level of knowledge about the care of the airway. The practice of airway care was also excellent level by $50 \%$. Conclusions: According to the statistical test Chi square test, significant relationship was found between the level of knowledge and practice of airway care performed by nurses in critical patients with endotracheal tube at Clínica Good Hope ( $\mathrm{p}$ value =, 000). Keywords: Knowledge, practice, airway care, patients, gold tracheal tube.
\end{abstract}

Keywords: Factor risk, urinary retention, immediate operated post.

*Correspondencia: bertha.gamboaupeu@gmail.com, 51-1-997515209 


\section{INTRODUCCIÓN}

A nivel nacional, según el Ministerio de Salud, las infecciones respiratorias intrahospitalarias constituyen una de las causas más importantes de morbimortalidad, representando la tasa de la mortalidad $12.08 \%$, para el año 2010.

El tubo orotraqueal (TOT) es la vía aérea más utilizada en asistencia ventilatoria invasiva, porque permite asegurar la oxigenación y mantener un medio para el drenaje de las secreciones, protegiendo a la persona de la asfixia. Según Sánchez (2007) esta alternativa representa, no obstante, una puerta de entrada para microorganismos y el cuidado del circuito ventilatorio, la cavidad oral y nasofaríngea del paciente, cobra especial importancia. Pulgarín, Osorio y Varela (2012) enfatizan la necesidad de personal competente que, valorando de modo crítico, integral y organizado, identifique con oportunidad los diagnósticos de enfermería o problemas del paciente $\mathrm{e}$ instaure rápidamente las medidas preventivas y correctivas, requiriendo en tal sentido de solidez, profundidad de conocimientos y metodología de trabajo. El profesional de enfermería, que labora en la unidad cuidados intensivos, realiza el manejo de la vía aérea en pacientes intubados, si no realiza una valoración adecuada al paciente ni el uso de las barreras protectoras, condiciona la ruptura de los mecanismos de defensa del huésped y el incremento de la colonización de microorganismos, posibilitando adquirir infecciones nosocomiales, por la frecuente aspiración de secreciones y el incumplimiento de las normas de asepsia.

Estas consideraciones llevaron a ejecutar el presente estudio, con el objetivo de determinar la relación existente entre el conocimiento y la práctica del cuidado de la vía aérea que realizan las enfermeras en las Unidades de Cuidados Intensivos y Cuidados Intermedios de la Clínica Good Hope.

\section{MATERIAL Y MÉTODOS}

La investigación realizada fue observacional, descriptiva, de corte transversal y diseño correlacional. La muestra fue equivalente al tamaño de la población $(n=30)$, dado que se ejecutó en una entidad privada de salud: Clínica Good Hope.

En la medición del nivel de conocimientos, el instrumento fue un cuestionario de 20 preguntas y la práctica del cuidado de la vía aérea se realizó mediante una guía de observación de 25 criterios, aplicada en dos oportunidades, en un lapso de 7 días, de domingo a viernes en turnos de mañana y tarde.

Ambos instrumentos fueron sometidos al juicio de expertos y la confiabilidad se estableció mediante el coeficiente alfa de Cronbach (0.873). Se realizó durante los meses de febrero a junio del 2012, con las enfermeras de los servicios de las unidades de cuidados intensivos e intermedios de la Clínica Good Hope.

Antes de la recolección de datos, se solicitó la autorización oficial para ejecutar el proyecto y previa coordinación con la jefatura de enfermería, se solicitó la aceptación de las enfermeras para la realización del estudio, previa información de riesgos y beneficios; en todo momento se respetó la autonomía del informante y la confidencialidad de la información mediante el anonimato

\section{RESULTADOS}

Tabla 1

Enfermeras según nivel de conocimiento en el cuidado de la vía aérea que realizan las enfermeras en pacientes críticos con tubo orotraqueal en la Clínica Good Hope. Lima, 2012.

\begin{tabular}{ccc}
\hline Nivel de conocimiento & $\mathrm{N}$ & $\%$ \\
\hline Excelente & 15 & 50.0 \\
Bueno & 8 & 26,7 \\
Regular & 7 & 23,3 \\
\hline Total & 30 & 100.0
\end{tabular}

Se puede apreciar, en la tabla 1, que del $100 \%$ de enfermeras del estudio, predomina en un $50 \%$ el conocimiento excelente, seguido del bueno y regular, con $26,7 \%$ y $23,3 \%$, respectivamente. 
Tabla 2

Enfermeras según nivel de práctica en el cuidado de la vía aérea que realizan las enfermeras en pacientes críticos con tubo orotraqueal en la clínica Good Hope. Lima, 2012.

\begin{tabular}{ccc}
\hline Nivel de práctica & $\mathrm{N}$ & $\%$ \\
\hline Excelente & 15 & 50.0 \\
Bueno & 12 & 40.0 \\
Regular & 3 & 10.0 \\
\hline Total & 30 & 100.0
\end{tabular}

En la tabla 2 se evidencia que del $100 \%$ de las enfermeras de la muestra estudiada, el 50\% alcanzó un nivel excelente de prácticas en el cuidado de la vía aérea, seguido del nivel bueno y regular, con $40,0 \%$ y $10,0 \%$, respectivamente.

Tabla 3

Relación entre el nivel de conocimiento y la práctica del cuidado de la vía aérea que realizan las enfermeras en pacientes críticos con tubo orotraqueal en la Clínica Good Hope. Lima 2012.

\begin{tabular}{cccc}
\hline Variable & Valor & gl & $\begin{array}{c}\text { Sig. asintótica } \\
\text { (bilateral) }\end{array}$ \\
\hline $\begin{array}{c}\text { Nivel de } \\
\text { conocimiento y la } \\
\text { práctica del cuidado }\end{array}$ & 23,693 & 4 &, 000 \\
\hline
\end{tabular}

De acuerdo a la tabla 3, la correlación según la prueba Chi cuadrado modificada de Pearson, fue significativa, siendo $p<0.05$; por tanto, existe relación entre las variables de nivel de conocimiento y práctica del cuidado de la vía aérea que realizan las enfermeras en pacientes críticos con tubo orotraqueal.

\section{DISCUSIÓN}

El $30 \%$ de las enfermeras, que trabajan en la Unidad de Cuidados Intensivos, tiene un tiempo de servicio menos de dos años, este grupo posee menos experiencia en relación a aquellas que tienen más de dos años de labor profesional en la especialidad. Acreditan un nivel de conocimiento bueno y aún no poseen la experiencia suficiente para el buen desempeño en el manejo de la vía aérea; al respecto, Narciso (2010) menciona que la práctica es sinónimo de experiencia, para que el ser humano ponga en práctica cierto tipo de conocimientos, sea este científico o vulgar, es necesario en primera instancia un primer acercamiento para el contacto directo mediante el uso de sentidos y conducta psicomotriz, es decir el experimento; no puede haber práctica sin experiencia previa.

Arteaga, Castro A., Castro J., y Solórzano (2010), concluyen que existe un elevado porcentaje de pacientes intubados en el área de Unidad de Cuidados
Intensivos y alta incidencia de infección nosocomial por la bacteria Pseudomona Aeruginosa, relacionado a la constante manipulación de limpieza de la vía aérea y al incorrecto manejo de este procedimiento.

Al respecto, según Pulgarín, Osorio y Varela (2012), señalan que la enfermera con conocimientos del riesgo existente, está preparada para reducir la alta incidencia de infección nosocomial, dentro de sus competencias procedimentales como el adecuado manejo de la vía aérea. Cabe destacar que es necesario además trasferir dichos conocimientos en una práctica concienzuda, por lo general el paciente crítico depende casi en su totalidad del cuidado enfermero; los pacientes intubados representan una condición de alta vulnerabilidad, no pueden expresar lo que sienten, ni hacer por sí mismos cuanto necesitan.

Benavente et al (2009) precisan que son más necesarios los cuidados de suplencia, la enfermera competente organiza su trabajo de manera que logra simultáneamente mantener la permeabilidad de las vías aéreas, favorecer una adecuada ventilación, sin agregar daños al paciente; comportamiento de cuidado que equivale, según Donabedian, a maximizar los beneficios para el paciente sin aumentar paralelamente los riesgos.

Los resultados del estudio expresan satisfactoriamente niveles de conocimiento y práctica 
de la vía aérea del $50 \%$ como excelentes, esto demuestra que el personal de enfermería que labora en la Clínica Good Hope, en las áreas de Cuidados Intensivos e Intermedios, se encuentra capacitado para desempeñarse ante pacientes críticos. Al respecto, Peña, Guevara y Delgado (2010), mencionan que en especialidades relevantes como cuidados intensivos, el rol de la enfermera exige que su perfil de desempeño evidencie mayor dominio teórico-práctico coherente con las demandas de los pacientes en estado crítico, quienes requieren de ellas (os) un alto compromiso por el nivel de dependencia que su estado de salud amerita.

La proyección de los resultados, según Urden, Lough y Stacy (1998), es favorable para los pacientes: la estancia será reducida, los costos se pueden optimizar y sobre todo la familia y el propio paciente experimentarán serenidad y satisfacción al ver que progresan paulatinamente con el apoyo de personal humanizado y altamente calificado para el cuidado crítico.

Un aspecto extraíble de estos resultados está dado por el significado que tienen como fuente de referencia para profesionales de entidades privadas, con demanda similar e intramuros. Constituye a su vez, un inventario de potencial humano apto para desarrollar procesos de socialización adecuados, con el personal nuevo que se incorpore a la Unidad de Cuidados Intensivos, como parte de las actividades de inducción.

Finalmente, en relación a nuestro objetivo de estudio, concluimos que existe relación significativa entre el nivel de conocimiento y la práctica del cuidado de la vía aérea que realizan las enfermeras en pacientes críticos con tubo orotraqueal en la Clínica Good Hope, esto evidencia que la enfermera tiene un perfil de desempeño de dominio teórico-práctico coherente en el cuidado de los pacientes en estado crítico.

\section{CONCLUSIONES}

El nivel de conocimiento de las enfermeras sobre el cuidado de la vía aérea en pacientes críticos con tubo orotraqueal, fue del $50 \%$ en el nivel excelente, $26,7 \%$ bueno. La práctica del cuidado de la vía aérea fue excelente en un $50 \%$, y el otro $50 \%$ entre bueno y regular, en el servicio de Unidad de Cuidados Intensivos de la Clínica Good Hope. Según la prueba estadística del Chi cuadrado modificado de Pearson, se encontró relación significativa entre ambas variables (valor $p=, 000)$.

\section{REFERENCIAS BIBLIOGRÁFICAS}

Arteaga, L. F., Castro J. A., Castro, A. L. \& Solórzano, J.B. (2010), Fortalecimiento de la investigación clínica epidemiológica en infecciones nosocomiales en pacientes sometidos a ventilación mecánica del área de UCI del hospital Verdi Cevallos Balda de Portoviejo de Mayo a Octubre. Tesis para la obtención de Médico Cirujano, Facultad de Ciencias de la Salud carrera Medicina, Universidad Técnica de Manabí, Portoviejo, Ecuador.

Benavente et al (2009). Fundamentos de enfermería. Editorial Mc-Graw Hill. Madrid.

Narciso, R. D. (2010). Nivel de conocimiento y práctica que tiene el enfermero (a) sobre la aspiración de secreciones en pacientes con intubación endotraqueal en el Servicio de Emergencia del Hospital Vitarte. Tesis para optar el grado Especialista en Enfermería de Emergencias y Desastres, Universidad Nacional Mayor de San Marcos, Lima, Perú.
Peña, M., Guevara, B. \& Delgado, C. (2010). Conocimiento que poseen las enfermeras intensivistas sobre el cuidado al paciente poli traumatizado con soporte ventilatorio antes y después de participar en un programa educativo teórico - práctico. Tesis Doctoral. Acarigua-Araure Estado Portuguesa- Venezuela.

Pulgarín, A., Osorio, S. \& Varela, L. (2012). Cuidado del paciente en estado crítico. Colombia: Corporación para Investigaciones Biológicas.

Sánchez, R. (2007). Atención especializada de enfermería al paciente ingresado en cuidados intensivos. Alcalá, España. $2^{\mathrm{a}}$ Edición.

Urden, Lough \& Stacy. (1998). Cuidados intensivos en enfermería, Barcelona, España. vol. 1 y 2. 3ra. Edición. Editorial Harcourt/océano. 